\title{
Approximations to the Stochastic Burgers Equation
}

\author{
Martin Hairer, Jochen Voss
}

24th May 2010

\begin{abstract}
This article is devoted to the numerical study of various finite difference approximations to the stochastic Burgers equation. Of particular interest in the one-dimensional case is the situation where the driving noise is white both in space and in time. We demonstrate that in this case, different finite difference schemes converge to different limiting processes as the mesh size tends to zero. A theoretical explanation of this phenomenon is given and we formulate a number of conjectures for more general classes of equations, supported by numerical evidence.
\end{abstract}

Keywords: stochastic Burgers equation, correction term, numerical approximation Subject classification: $60 \mathrm{H} 35,60 \mathrm{H} 15,35 \mathrm{~K} 55$

\section{Introduction}

This article studies several finite difference schemes for the viscous stochastic Burgers equation:

$$
\partial_{t} u(x, t)=\nu \partial_{x}^{2} u-g(u) \partial_{x} u+\sigma \xi(x, t), \quad x \in[0,2 \pi], \quad t \geq 0 .
$$

In this equation, $\xi$ denotes space-time white noise, that is the centred, distribution-valued Gaussian random variable such that $\mathbb{E}(\xi(x, t) \xi(y, s))=\delta(t-s) \delta(x-y)$. We will always endow this equation with periodic boundary conditions and we will consider solutions $u$ taking values either in $\mathbb{R}$ or in $\mathbb{R}^{n}$ (in which case $g$ is matrix-valued in general).

Motivations for studying the stochastic Burgers equation are manifold. Just to name a few, it is used to model vortex lines in high-temperature superconductors $\mathrm{BFG}^{+} 94$, dislocations in disordered solids and kinetic roughening of interfaces in epitaxial growth Bar96, formation of large-scale structures in the universe GSS85, SZ89, constructive quantum field theory [BCJL94, etc. Since in the case $\sigma=0$ and $g(u) \propto u$ this equation is furthermore explicitly solvable via the Hopf-Cole transform $\left(u=\partial_{x} v / v\right.$, where $v$ solves the heat equation), it comes as no surprise that a wealth of numerical and analytical results are available. From a purely mathematical point of view, let us mention for example the well-posedness results from BCF91, BCJL94, DPDT94, Gyö98, Kim06 and the ergodicity results obtained in TZ06, GM05. One remarkable achievement was the construction of a stationary solution in the inviscid limit with non-vanishing noise EKMS00] (dissipation then occurs purely through shocks). From a more quantitative perspective, the scaling exponents of the solutions in the small viscosity limit have attracted considerable interest, both in the physics and the applied mathematics literature [YC96, BM96. Kra99, EVE00a, EVE00b.

The white noise term $\xi$ in (1D) leads to solutions $u$ which, in general, will be very "rough". In particular, $u$ as a function of the spatial variable $x$ will not be differentiable in the classical sense, but will only possess some Hölder regularity. As a consequence, it transpires that the solutions to (11) are extremely unstable under natural approximations of the nonlinearity, and this is the phenomenon that will be explored in this article. For example, for any $a, b \geq 0$ with $a+b>0$, one can consider the approximating equation

$$
\partial_{t} u^{\varepsilon}(x, t)=\nu \partial_{x}^{2} u^{\varepsilon}-g\left(u^{\varepsilon}\right) D_{\varepsilon} u^{\varepsilon}(x, t)+\sigma \xi(x, t),
$$

where the approximate derivative $D_{\varepsilon}$ is defined as

$$
D_{\varepsilon} u(x, t)=\frac{u(x+a \varepsilon, t)-u(x-b \varepsilon, t)}{(a+b) \varepsilon} .
$$


In the absence of the noise term $\xi$ it would be a standard exercise in numerical analysis to show that the solution of (2) converges to the solution of (1) as $\varepsilon \rightarrow 0$. This is just an example of the widely accepted 'folklore' fact that, if an equation is well-posed, any 'reasonable' approximation will converge to the exact solution 1

In this article, we will argue that, if $\xi$ is taken to be space-time white noise, the limit of (2) as $\varepsilon \rightarrow 0$ depends on the values $a$ and $b$ and is equal to (11) only if either $g$ is constant or $a=b$ ! Furthermore, it will follow from the argument that, if one considers driving noise that is slightly rougher than space-time white noise (taking a noise term equal to $\left(1-\partial_{x}^{2}\right)^{\alpha} d w(t)$ with $\alpha \in(0,1 / 4)$ still yields a well-posed equation), one does not expect solutions to the approximate equation (2) to converge to anything at all, unless $a=b$. Our methodology here is to first present a heuristic argument which allows to derive quantitative predictions for the effect of the finite difference discretisation on the solution. We will then use numerical experiments to verify these predictions.

At this point we would like to emphasise that the aim of this article is certainly not to advocate the use of a finite difference scheme of the type (2) to effectively simulate (11). Indeed, we will show in section 2.2 below that approximations of the nonlinearity of the type $D_{\varepsilon} G(u)$, where $G$ is the antiderivative of $g$ already have much better stability properties. Instead, our aim is merely to give a striking illustration of the fact that caution should be exercised in the simulation of stochastic PDEs driven by spatially rough noise.

The text is structured as follows: We start in section 2 by presenting our argument for the case of the stochastic Burgers equation, i.e. for $g(u)=u$. In section 3 we will present the corresponding results for more general equations and in section 4 we study the limit of vanishing noise and viscosity. Finally, in appendix $\mathrm{A}$ we discuss some technical aspects of the simulations used throughout this article.

\section{Acknowledgements}

We would like to thank Andrew Majda, Andrew Stuart, Eric Vanden Eijnden, and Sam Falle for helpful discussions of the phenomena discussed in this article. Financial support was kindly provided by the EPSRC through grants EP/E002269/1 and EP/D071593/1, as well as by the Royal Society through a Wolfson Research Merit Award. JV would like to thank the Courant Institute, where work on this article was started, for its hospitality.

\section{Stochastic Burgers Equation}

In this section we consider the stochastic Burgers equation

$$
d u=\nu \partial_{x}^{2} u d t-u \partial_{x} u d t+\sigma d w,
$$

as well as the approximation given by

$$
d u^{\varepsilon}=\nu \partial_{x}^{2} u^{\varepsilon} d t-u^{\varepsilon} D_{\varepsilon} u^{\varepsilon} d t+\sigma d w .
$$

The solutions $u$ and $u^{\varepsilon}$ take values in the space $L^{2}([0,2 \pi], \mathbb{R})$ on which the operator $\partial_{x}^{2}$ is endowed with periodic boundary conditions, and $w$ is an $L^{2}$-cylindrical Wiener process (see DPZ92 for details). Equation (4) is well-posed, since we can rewrite $u \partial_{x} u$ as $\frac{1}{2} \partial_{x}\left(u^{2}\right)$ which is locally Lipschitz from the Sobolev space $H^{1 / 4}$ into $H^{-1}$, thus allowing us to apply general local well-posedness theorems as in DPZ92, Hai09. For fixed time $t>0$, the solutions to (4) have the regularity of Brownian motion when viewed as a function of the spatial variable $x$. In particular, they are not differentiable in $x$. Figure 1 shows numerical solutions of equation (5) for different values of $a$ and $b$. One can see that different choices for these parameters lead to an $\mathcal{O}(1)$ difference in the solutions.

Our aim in this article is to understand and quantify these differences. In particular, in conjecture 1 below, we compute a correction term to (4) and we verify numerically that the solutions to (5) converge to the corrected equation as $\varepsilon \rightarrow 0$. This understanding will then allow us to conjecture the appearance of similar correction terms in more complicated situations and we will again verify these conjectures numerically.

\footnotetext{
${ }^{1}$ Remember that we are working at fixed non-zero viscosity here, so there is no ambiguity in the concept of solution and we do not require an upwind scheme in order to obtain convergence.
} 


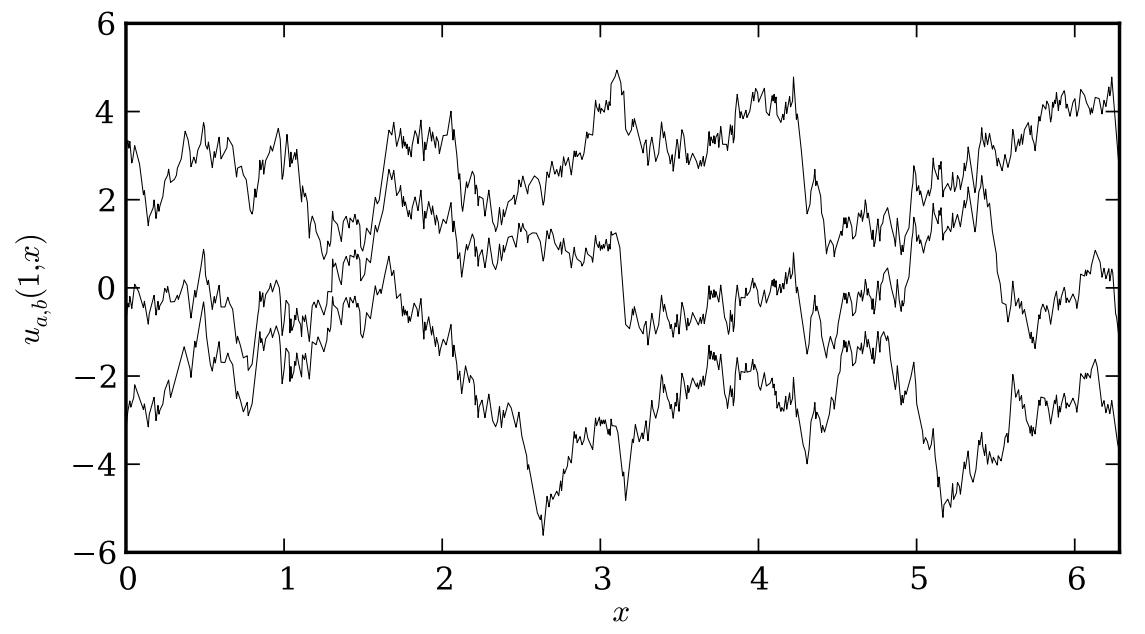

Figure 1: Illustration of the discretisation error for the finite difference method (5). The three lines correspond to a right-sided discretisation ( $a=1, b=0$; top-most curve), a centred discretisation ( $a=1, b=1$; middle curve) and a left-sided discretisation ( $a=0, b=1$; bottom-most curve), all computed using the same instance of the driving noise. The picture clearly shows that there is an $\mathcal{O}(1)$ difference between the solutions obtained by the three different discretisation schemes. From the argument presented in the text, we assume that the exact solution of (4) will be closest to the middle of the three lines.

\subsection{Heuristic Explanation}

For simplicity, instead of $u$ we consider the solution $v$ to the stochastic heat equation

$$
d v=\nu \partial_{x}^{2} v d t+\sigma d w(t)
$$

Since the properties of the discretisation of differential operators only depend on local properties, and since $v$ has the same spatial regularity as $u$, it will be sufficient to study how well $v D_{\varepsilon} v$ approximates $v \partial_{x} v=\frac{1}{2} \partial_{x} v^{2}$.

By expressing $v$ in the Fourier basis $\left\{\mathrm{e}^{i n x} / \sqrt{2 \pi}\right\}_{n \in \mathbb{Z}}$ it is easy to check that the stationary solution to (6) is

$$
v(t, x)=\sum_{n \in \mathbb{Z} \backslash\{0\}} \frac{\sigma}{i n \sqrt{2 \nu}} \xi_{n}(t) \frac{\mathrm{e}^{i n x}}{\sqrt{2 \pi}}+\xi_{0}(t) \frac{1}{\sqrt{2 \pi}},
$$

where the $\xi_{0}$ is a (real-valued) standard Brownian motion and $\xi_{n}$ for $n \neq 0$ are complex-valued Ornstein-Uhlenbeck processes with variance 1 (in the sense that $\mathbb{E}\left|\xi_{n}(t)\right|^{2}=1$ ) and time constant $\nu n^{2}$ which are independent, except for the condition that $\xi_{-n}=\bar{\xi}_{n}$. Therefore, the derivative of $v$ is given (at least formally) by

$$
\partial_{x} v(x)=\sum_{n \neq 0} \frac{\sigma \xi_{n}(t) \mathrm{e}^{i n x}}{2 \sqrt{\nu \pi}} .
$$

The $\varepsilon$-approximation to the derivative given in (3), on the other hand, is given by

$$
D_{\varepsilon} v(x)=\sum_{n \neq 0} \frac{\sigma \xi_{n}(t) \mathrm{e}^{i n x}}{2 \sqrt{\nu \pi}} \frac{\mathrm{e}^{i n a \varepsilon}-\mathrm{e}^{-i n b \varepsilon}}{(a+b) i \varepsilon n} .
$$

It is clear that the terms in (8) are a good approximation to the terms in (7) only up to $n \approx \varepsilon^{-1}$. For larger $n$, the multiplier in (8) will decrease like $n^{-1}$.

For our analysis we restrict ourselves to the constant $(n=0)$ Fourier mode. Our numerical experiments, below, show that the contributions from this mode are already enough to explain the observed differences between the solutions of the approximating equation (5) and the exact 
solution. Since $v \partial_{x} v$ is a total derivative, the 0 -mode of this term vanishes. In contrast, the 0 -mode of $v D_{\varepsilon} v$ does not vanish at all: We obtain instead for this term the sum

$$
\begin{aligned}
\left\langle v D_{\varepsilon} v \mid \frac{1}{\sqrt{2 \pi}}\right\rangle & =\frac{1}{\sqrt{2 \pi}} \sum_{n \neq 0} \frac{\sigma^{2} \xi_{-n}(t) \xi_{n}(t)}{2 \nu(-i n)} \frac{\mathrm{e}^{i n a \varepsilon}-\mathrm{e}^{-i n b \varepsilon}}{(a+b) \varepsilon i n} \\
& =\frac{\sigma^{2}}{\sqrt{2 \pi} \nu} \sum_{n>0}\left|\xi_{n}(t)\right|^{2} \frac{\cos a \varepsilon n-\cos b \varepsilon n}{(a+b) \varepsilon n^{2}}
\end{aligned}
$$

and the expectation of this expression, as $\varepsilon \rightarrow 0$, converges to

$$
\begin{aligned}
\lim _{\varepsilon \downarrow 0} \mathbb{E}\left(-v D_{\varepsilon} v\right)(x) & =\lim _{\varepsilon \downarrow 0} \mathbb{E}\left\langle-v D_{\varepsilon} v \mid \frac{1}{\sqrt{2 \pi}}\right\rangle \frac{1}{\sqrt{2 \pi}} \\
& =-\frac{\sigma^{2}}{2 \pi \nu} \int_{0}^{\infty} \frac{\cos a \kappa-\cos b \kappa}{(a+b) \kappa^{2}} d \kappa=\frac{\sigma^{2}}{4 \nu} \frac{a-b}{a+b} .
\end{aligned}
$$

As a consequence, one expects the following result.

Conjecture 1 The solution of the approximating equation (5) converges, as $\varepsilon \rightarrow 0$, to the solution of

$$
d u=\nu \partial_{x}^{2} u d t-u \partial_{x} u d t+\frac{\sigma^{2}}{4 \nu} \frac{a-b}{a+b} d t+\sigma d w .
$$

Thus, the approximation converges to the stochastic Burgers equation (4) only for $a=b$.

Remark 2.1 The solution to the stochastic Burgers equation (or rather the integrated process which solves the corresponding KPZ equation) arising as the fluctuations process in the weakly asymmetric exclusion process [BG97, BQS09] is driven by the derivative of space-time white noise. As a consequence, it does not solve an SPDE that is well-posed in the classical sense and can currently only be defined via the Hopf-Cole transform. Such a process is even rougher (by "one derivative") than the process considered here and one would expect the "wrong' numerical approximation schemes to fail there in an even more spectacular way.

Remark 2.2 One may think of two reasons why the correction term vanishes when $a=b$. On the one hand, this discretisation is more symmetric. On the other hand, it yields a second-order approximation to the derivative at $x$. The correct explanation is closer to the first one. Indeed, consider for example the discretisation

$$
\left(\tilde{D}_{\varepsilon} u\right)(x) \approx \frac{c u(x+2 \varepsilon)+(1-3 c) u(x+\varepsilon)+3 c u(x)-(1+c) u(x-\varepsilon)}{2 \varepsilon} .
$$

This discretisation is of second order for every $c \in \mathbb{R}$. However, if we perform the same calculation as above with this discretisation, we obtain a correction term equal to

$$
\frac{\sigma^{2}}{2 \pi \nu} \int_{0}^{\infty} \frac{c \cos 2 \kappa-4 c \cos \kappa+3 c}{2 \kappa^{2}} d \kappa=-\frac{c \sigma^{2}}{8 \nu}
$$

which vanishes only if $c=0$, i.e. when (11) conincides with the symmetric discretisation (3).

In the above calculation, both the limiting equation and the approximating equation live in the same space. It is possible to carry out a similar analysis in the case where the approximating equation takes values in a different space, typically $\mathbb{R}^{N}$ for some large $N$. For example, we can consider the 'finite difference' approximation given by

$$
\begin{gathered}
\partial_{x}^{2} u \approx \frac{u(x+\delta)-2 u(x)+u(x-\delta)}{\delta^{2}} \stackrel{\text { def }}{=}\left(\Delta_{N} u\right)(x) \\
u \partial_{x} u \approx u D_{\delta} u=u(x) \frac{u(x+\delta)-u(x)}{\delta} \stackrel{\text { def }}{=} F_{N}(u)(x),
\end{gathered}
$$

where we set $\delta=\frac{2 \pi}{N}$ for the approximation with $N$ gridpoints. In this setting, we approximate $u$ by $u^{N} \in \mathbb{R}^{N}$ with $u_{j}^{N} \approx u(j \delta)$. The natural candidate for the approximation of space-time 
white noise is then given by $d W_{j}^{N}=\delta^{-1 / 2} d W_{j}$, where the $W_{j}$ are independent, one-dimensional standard Brownian motions. This is the correct scaling, since it ensures for example that for every continuous function $g, \sum_{j} W_{j}^{N} g(\delta j)$ is a Wiener process with covariance $\sum_{j} g(\delta j)^{2} \delta \approx \int g^{2} d x$. With this notation, we consider the approximating equation given by

$$
d u^{N}=\nu \Delta_{N} u^{N} d t-F_{N}\left(u^{N}\right) d t+\sigma d W^{N}(t) .
$$

Let us take $N$ even for the sake of simplicity. It is then straightforward to check that the eigenvectors for $\Delta_{N}$ are given as before by $\mathrm{e}^{i n x}$ with $n=-\frac{N}{2}+1, \ldots, \frac{N}{2}$, but the corresponding eigenvalues are

$$
\lambda_{n}=\frac{2}{\delta^{2}}(\cos n \delta-1)=-\left(\frac{2}{\delta} \sin \left(\frac{n \delta}{2}\right)\right)^{2} \stackrel{\text { def }}{=}-\eta_{n}^{2} .
$$

(Note that for fixed $n$ and small $\delta$, one has indeed $\lambda_{n} \approx-n^{2}$.) It then follows as previously that the solution to the linearised equation is given by

$$
v(t, x)=\sum_{n \neq 0} \frac{\sigma}{2 \sqrt{\nu \pi} i \eta_{n}} \mathrm{e}^{i n x} \xi_{n}(t)+\xi_{0}(t) \frac{1}{\sqrt{2 \pi}}
$$

and that its discrete derivative $D_{\delta} v$ is given by

$$
D_{\delta} v(t, x)=\sum_{n \neq 0} \frac{\sigma \mathrm{e}^{i n x} \xi_{n}(t)}{2 \sqrt{\nu \pi} i \eta_{n}} \frac{\mathrm{e}^{i n \delta}-1}{\delta} .
$$

Note that the sums in both expressions only run over the values $n=-\frac{N}{2}+1, \ldots, \frac{N}{2}$. Similarly to above, we obtain that the expectation of the zero mode of the product $v D_{\delta} v$ is given by

$$
\mathbb{E}\left\langle-v D_{\varepsilon} v \mid \frac{1}{\sqrt{2 \pi}}\right\rangle \frac{1}{\sqrt{2 \pi}}=-\frac{\sigma^{2}}{2 \pi \nu} \sum_{n=1}^{N / 2} \frac{\cos \delta n-1}{\delta \eta_{n}^{2}}=\frac{\sigma^{2}}{2 \pi \nu} \sum_{n=1}^{N / 2} \frac{\delta}{2}=\frac{\sigma^{2}}{4 \nu} .
$$

One therefore expects the following result.

Conjecture 2 The solution $u^{N}$ of the finite difference approximation (13) converges, as $N \rightarrow$ $\infty$, to the solution of

$$
d u=\nu \partial_{x}^{2} u d t-u \partial_{x} u d t+\frac{\sigma^{2}}{4 \nu} d t+\sigma d w(t)
$$

To test this conjecture, we use the following numerical experiment: We numerically solve both the "approximating" equation (13) and the "corrected" SPDE

$$
d u_{\gamma}=\nu \partial_{x}^{2} u_{\gamma} d t-u_{\gamma} \partial_{x} u_{\gamma} d t+\gamma \frac{\sigma^{2}}{\nu} d t+\sigma d w(t)
$$

until a fixed time $T$, using the same instance of the noise. To discretise the term $u \partial_{x} u$ in (15) we use the approximation $\left(u(x)^{2} / 2-u(x-\delta)^{2} / 2\right) / \delta$, which is known to converge to the exact solution, see section 2.2 below. Also, for increased accuracy, we use a finer grid for (15) than we did for (13). Now we can compare the two solutions by considering $\left\|u^{N}(T, \cdot)-u_{\gamma}(T, \cdot)\right\|_{2}$ as a function of $\gamma$ : If conjecture 2 is correct, this function will take its minimum at $\gamma \approx 1 / 4$. The result of such a simulation is given by the line labelled "finite difference" in figure 2, The minimum of the curve is indeed located close to $\gamma=1 / 4$, thus giving support to conjecture 2 .

Even though the correction terms in conjectures 1 and 2 (with $a=1$ and $b=0$ ) coincide, the constants arise in completely different ways. This might lead to the speculation that the value of this constant is an intrinsic property of the limiting equation, rather than of the particular way of approximating it. The following argument shows that this is not the case. One can repeat the calculation leading to conjecture 2 with a 'spectral Galerkin' approximation of the linear part of the equation, but retaining a 'finite difference' approximation of the nonlinearity. In other words, we consider (13) as before, but we take for $\Delta_{N}$ the self-adjoint matrix with eigenvectors $\mathrm{e}^{\text {inx }}$ and eigenvalues $-n^{2}$. (This can be achieved by first applying the discrete Fourier transform, then 


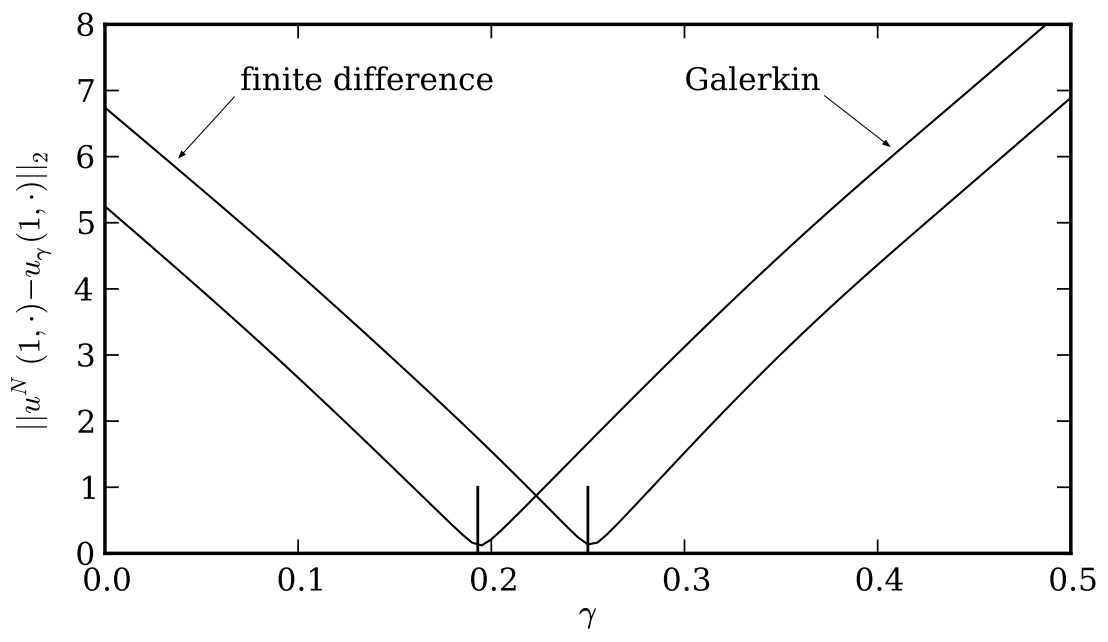

Figure 2: This figure compares the solution $u^{N}$ of the finite difference approximation (13) to the solution $u_{\gamma}$ of the "corrected" SPDE (15) (which includes an additional drift term $\gamma \sigma^{2} / \nu$ ). The curves show the $L^{2}$-norm difference between the solutions (for the same instance of the noise) as a function of $\gamma$, once using finite difference discretisation (12) of the linear part (corresponding to the top-most curve in figure 1) and once using the spectral Galerkin discretisation. The two vertical line segments give the predicted locations for the minima of the two curves. It can be seen that predictions and simulations are in good agreement.

multiplying the $n$th component by $-n^{2}$, and finally applying the inverse Fourier transform.) In this case one has $\eta_{n}=n$ and it transpires that the correction term is given by

$$
\sum_{n=1}^{N / 2} \frac{\sigma^{2}}{2 \pi \nu \eta_{n}^{2}} \frac{1-\cos n \delta}{\delta} \approx \frac{\sigma^{2}}{2 \pi \nu} \int_{0}^{\pi} \frac{1-\cos \kappa}{\kappa^{2}} d \kappa \approx \frac{0.193 \sigma^{2}}{\nu} .
$$

which is clearly different from (14).

To verify that the spectral Galerkin discretisation of the linear part indeed leads to this different correction term, we modify the code which we used to validate conjecture 2 above. The result of this simulation is given by the line labelled "Galerkin" in figure 2 Again, there is good agreement between our conjecture and the simulation results.

\subsection{The Case of More Regular Noise}

To conclude this section, let us argue that the situation considered in this article is truly a borderline case in terms of regularity and that if we drive (5) by noise that gives rise to slightly more regular solutions, the solutions of (5) converge to those of (4) without any correction term. Indeed, consider a general semilinear stochastic PDE driven by additive noise:

$$
d u=-A u d t+F(u) d t+Q d w(t),
$$

where $A$ is a strictly positive-definite selfadjoint operator on some Hilbert space $\mathcal{H}, F$ is a (possibly unbounded) nonlinear operator from $\mathcal{H}$ to $\mathcal{H}, W$ is a standard cylindrical Wiener process on $\mathcal{H}$, and $Q: \mathcal{H} \rightarrow \mathcal{H}$ is a bounded operator.

Denote furthermore $\mathcal{H}^{\alpha}=\mathcal{D}\left(A^{\alpha}\right)$ for $\alpha>0$ and let $\mathcal{H}^{-\alpha}$ be the dual space to $\mathcal{H}^{\alpha}$ under the dual pairing given by the Hilbert space structure of $\mathcal{H}$. (So that $\mathcal{H}^{-\alpha}$ is a superspace of $\mathcal{H}$ for $\alpha>0$.) We also denote by $\|\cdot\|_{\alpha}$ the natural norm of $\mathcal{H}^{\alpha}$. Finally, we denote as before by $v$ the solution to the linearised system

$$
d v=-A v d t+Q d w(t),
$$

which we assume to be an $\mathcal{H}$-valued Gaussian process with almost surely continuous sample paths. One then has the following convergence result: 
Theorem 2.3 Assume that there exists $\gamma \geq 0$ and $a \in[0,1)$ such that $F: \mathcal{H}^{\gamma} \rightarrow \mathcal{H}^{\gamma-a}$ is locally Lipschitz continuous and such that the process $v$ has continuous sample paths with values in $\mathcal{H}^{\gamma}$. Assume furthermore that $F_{\varepsilon}: \mathcal{H}^{\gamma} \rightarrow \mathcal{H}^{\gamma-a}$ is such that $F_{\varepsilon}$ is locally Lipschitz and such that the convergence $F_{\varepsilon}(u) \rightarrow F(u)$ takes place in $\mathcal{H}^{\gamma-a}$, locally uniformly in $\mathcal{H}^{\gamma}$. Then, the solutions $u_{\varepsilon}$ to

$$
d u_{\varepsilon}=-A u_{\varepsilon} d t+F\left(u_{\varepsilon}\right) d t+Q d w(t),
$$

converge to the solutions to (16) as $\varepsilon \rightarrow 0$.

Proof. The proof is straightforward and we only sketch it. We assume without loss of generality that the parameter $\varepsilon$ is chosen in such a way that for every $R>0$ there exists a constant $C_{R}$ such that

$$
\sup _{\|x\|_{\gamma} \leq R}\left\|F_{\varepsilon}(u)-F(u)\right\|_{\gamma-a} \leq C_{R} \varepsilon .
$$

Denote now by $u$ the solution to (16) with initial condition $u_{0}$ and by $u_{\varepsilon}$ the solution to (17) with initial condition $u_{0}^{\varepsilon}$. Let $t>0$ be small enough so that $\|u(s)\|_{\gamma} \leq R$ and $\left\|u(s)-u_{\varepsilon}(s)\right\|_{\gamma} \leq R$ for $s \leq t$. We then have

$$
\begin{aligned}
\left\|u(t)-u_{\varepsilon}(t)\right\|_{\gamma} \leq\left\|u_{0}-u_{0}^{\varepsilon}\right\|_{\gamma}+C \int_{0}^{t}(t-s)^{-a}\left\|F_{\varepsilon}\left(u_{\varepsilon}(s)\right)-F(u(s))\right\|_{\gamma-a} d s \\
\leq\left\|u_{0}-u_{0}^{\varepsilon}\right\|_{\gamma}+C \int_{0}^{t}(t-s)^{-a}\left\|F_{\varepsilon}\left(u_{\varepsilon}(s)\right)-F\left(u_{\varepsilon}(s)\right)\right\|_{\gamma-a} d s \\
\quad+C \int_{0}^{t}(t-s)^{-a}\left\|F\left(u_{\varepsilon}(s)\right)-F(u(s))\right\|_{\gamma-a} d s \\
\leq\left\|u_{0}-u_{0}^{\varepsilon}\right\|_{\gamma}+C_{R} \varepsilon+C_{R} t^{1-a} \sup _{s \leq t}\left\|u(s)-u_{\varepsilon}(s)\right\|_{\gamma} .
\end{aligned}
$$

The claim then follows by taking $t$ sufficiently small and performing a simple iteration.

Despite its simplicity, this criterion is surprisingly sharp. Indeed, we argue that if we consider (44) but with the space-time white noise $d w$ replaced by $\left(1-\partial_{x}^{2}\right)^{-\delta} d w$ for $\delta>0$, then the assumptions of theorem 2.3 can be satisfied with some choice of exponent $\gamma$ for the approximation

$$
F_{\varepsilon}(u)(x)=u(x) \frac{u(x+\varepsilon)-u(x)}{\varepsilon} \stackrel{\text { def }}{=} u(x)\left(D_{\varepsilon} u\right)(x) .
$$

Obviously, this cannot be the case when $\delta=0$, since we then observe the convergence to solutions to (10).

Indeed, we first note that since the linear operator appearing in (4) is of second order, we have the correspondence

$$
\mathcal{H}^{\gamma}=H^{2 \gamma},
$$

between interpolation spaces and fractional Sobolev spaces. In order to keep our notation coherent throughout this section, we still denote by $\|\cdot\|_{\gamma}$ the norm of $\mathcal{H}^{\gamma}$, i.e. $\|u\|_{\gamma}=\left\|\left(1-\partial_{x}^{2}\right)^{\gamma} u\right\|_{L^{2}}$, where we implicitly endow $\partial_{x}^{2}$ with periodic boundary conditions. With this notation, we get the following result.

Lemma 2.4 For $\gamma \geq 0$ and $a \in\left[\frac{1}{2}, 1\right]$, we have $\left\|D_{\varepsilon} u-\partial_{x} u\right\|_{\gamma-a} \leq C \varepsilon^{2 a-1}\|u\|_{\gamma}$.

Proof. The operator $D_{\varepsilon}-\partial_{x}$ is given by the Fourier multiplier $M_{\varepsilon}(k)=\varepsilon^{-1}\left(e^{i k \varepsilon}-1-i k \varepsilon\right)$. We immediately obtain the bound

$$
\left|M_{\varepsilon}(k)\right| \leq k(\varepsilon k \wedge 1) \leq k(\varepsilon k)^{2 a-1},
$$

from which the claim follows at once.

Since furthermore $\mathcal{H}^{\gamma}$ is an algebra for $\gamma>\frac{1}{4}$, we conclude that the bound (18) holds (for some different power of $\varepsilon$ ), provided that we choose $\gamma \in\left(\frac{1}{4}, \frac{1}{2}\right]$ and $a=2 \gamma$. On the other hand, the solution to the linearised equation

$$
d v=\partial_{x}^{2} v d t+\left(1-\partial_{x}^{2}\right)^{-\delta} d w
$$




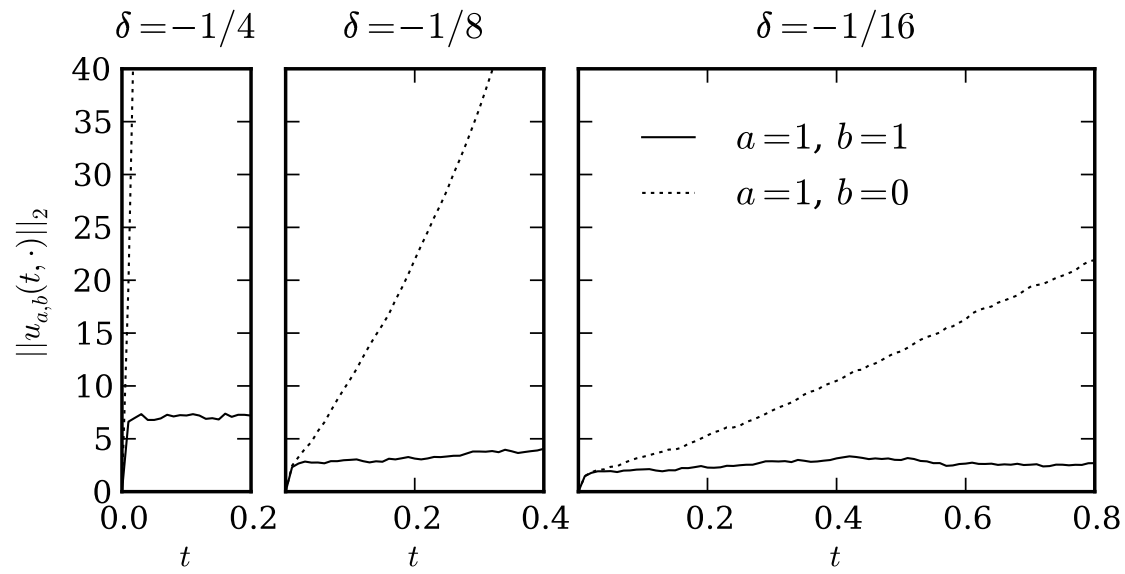

Figure 3: Illustration of the divergence of the right-sided discretisation for noise rougher than space-time white noise. The three panels show, for different values of $\delta$, the $L^{2}$-norm of numerical solutions to equation (20). The full lines correspond to a centred discretisation $(a=1, b=1)$ whereas the dotted lines correspond to the right-sided discretisation $(a=1, b=0)$. The figures show that only the centred discretisation seems to be stable for $\delta<0$.

belongs to $\mathcal{H}^{\gamma}$ if and only if $\gamma<\frac{1}{4}+\delta$, thus supporting our claim that the case $\delta=0$ is precisely borderline for the applicability of theorem 2.3 .

If on the other hand we make the more natural choice

$$
F_{\varepsilon}(u)=\frac{1}{2} D_{\varepsilon}\left(u^{2}\right)
$$

then it turns out that we can apply theorem 2.3 even in the case $\delta=0$. Indeed, it follows from standard Sobolev theory (see for example [Hai09]) that if $\gamma \in\left(\frac{1}{8}, \frac{1}{4}\right)$, the map $u \mapsto u^{2}$ is locally Lipschitz from $\mathcal{H}^{\gamma}$ into $\mathcal{H}^{\beta}$ provided that $\beta<2 \gamma-\frac{1}{4}$. As a consequence, it follows from lemma 2.4 that the approximation $F_{\varepsilon}$ given by (19) converges to $u \partial_{x} u$ in the sense of (18), provided that $\gamma \in\left(\frac{1}{8}, \frac{1}{4}\right)$ and $a \in\left(\frac{3}{4}-\gamma, 1\right)$.

Remark 2.5 Some ad hoc numerical scheme was shown to converge to the exact solution in AG06. Some other schemes are shown to converge in GKN02, but only in the case $\delta>0$ of more regular noise. A particle approximation to a specific modification was constructed in GD04.

\subsection{Numerical Verification of the Borderline Case}

We have performed numerical simulations that corroborate the argument presented in the previous section and show that $\delta=0$ truly is the borderline case for the limiting equation to be independent on the type of discretisation performed on the nonlinearity. In the case $\delta<0$ (i.e. the case where the driving noise is rougher than space-time white noise), our preceding discussion suggests that the centred discretisation should converge to the correct solution for $|\delta|$ sufficiently small, but that the solutions to both the left-sided and the right-sided discretisations should diverge as $\varepsilon \rightarrow 0$.

In order to verify this effect, we numerically solve the SPDE

$$
d u=\nu \partial_{x}^{2} u d t-u \partial_{x} u d t+\left(1-\partial_{x}^{2}\right)^{-\delta} d w,
$$

using different discretisation schemes and different values for $\delta$. The results are shown in figure 3 , The simulations are in good agreement with the argument outlined above. 


\section{Possible Generalisations of the Argument}

In this section, we discuss a number of possible extensions of these results to more general Burgers-type equations. We restrict ourselves in this discussion to the case $a=1, b=0$, i.e. to right-sided discretisations. This is purely for notational convenience, and one would expect similar correction terms to appear for arbitrary values of $a$ and $b$, just as before.

\subsection{More General Nonlinearities}

Consider the equation

$$
d u_{i}=\nu \partial_{x}^{2} u_{i} d t+\sum_{j=1}^{d} \partial_{j} h_{i}(u) \partial_{x} u_{j} d t+\sigma d w_{i}
$$

for an $\mathbb{R}^{d}$-valued process $u$ and a smooth function $h: \mathbb{R}^{d} \rightarrow \mathbb{R}^{d}$ with bounded second and third derivatives. Rewriting the nonlinearity as $\partial_{x}\left(h_{i}(u)\right)$, we see that this equation is globally wellposed. As before, we consider the approximating equation

$$
d u_{i}^{\varepsilon}(x, t)=\nu \partial_{x}^{2} u_{i}^{\varepsilon}(x, t) d t+\sum_{j=1}^{d} \partial_{j} h_{i}\left(u^{\varepsilon}(x, t)\right) D_{\varepsilon} u_{j}^{\varepsilon}(x, t) d t+\sigma d w_{i}(t)
$$

The idea now is to introduce a cut-off frequency $N$ and to write $u^{\varepsilon}=\bar{u}^{\varepsilon}+\tilde{u}^{\varepsilon}$, where $\bar{u}^{\varepsilon}$ is the projection of $u^{\varepsilon}$ onto Fourier modes with $|k| \leq N$. Since the linear part of the equation dominates the nonlinearity at high frequencies, one expects $\tilde{u}^{\varepsilon}$ to be well approximated by $\tilde{v}$, the projection of $v$ onto the high frequencies. This on the other hand is small in the $L^{\infty}$ norm (it decreases like $N^{-s}$ for every $s<\frac{1}{2}$ ), so that

$$
\partial_{j} h_{i}\left(u^{\varepsilon}\right) \approx \partial_{j} h_{i}\left(\bar{u}^{\varepsilon}\right)+\sum_{k=1}^{d} \partial_{j k}^{2} h_{i}\left(\bar{u}^{\varepsilon}\right) \tilde{v}_{k} .
$$

It now follows from the same argument as before that the term $\partial_{j k}^{2} h_{i}\left(\bar{u}^{\varepsilon}\right) \tilde{v}_{k} D_{\varepsilon} v_{j}$ is expected to yield a non-vanishing contribution for $k=j$ in the limit $\varepsilon \rightarrow 0$ and $N \rightarrow \infty$. Provided that we keep $N \ll \frac{1}{\varepsilon}$, this contribution will again be described by (9), so that we expect the following behaviour:

Conjecture 3 The solution of (22) converges, as $\varepsilon \rightarrow 0$, to the solution of

$$
d u_{i}=\nu \partial_{x}^{2} u_{i} d t+\sum_{j}\left(\partial_{j} h_{i}(u) \partial_{x} u_{j}-\frac{\sigma^{2}}{4 \nu} \partial_{j j}^{2} h_{i}(u)\right) d t+\sigma d w_{i}
$$

In the one-dimensional case we can recover conjecture 1 (for $a=1, b=0$ ) from conjecture 3 by choosing $h(u)=-u^{2} / 2$ in (23).

We perform the following numerical experiment to validate the functional form of the correction term given in conjecture 3. We numerically solve both the "approximating" equation (22) and, for $p: \mathbb{R} \rightarrow \mathbb{R}$, the "corrected" SPDE

$$
d u_{p}=\nu \partial_{x}^{2} u_{p} d t+h^{\prime}\left(u_{p}\right) \partial_{x} u_{p} d t-\frac{\sigma^{2}}{4 \nu} p\left(u_{p}\right) d t+\sigma d w
$$

until a fixed time $T$, using the same instance of the noise. As for the discretisation of (15) above, we use the approximation $(h(x)-h(x-\delta)) / \delta$ for the term $h^{\prime}(u) \partial_{x} u$ in the proposed limit (24) and we also solve (24) on a finer grid than we use for (22). Finally, we numerically optimise the correction term $p$ (using some parametric form) in order to minimise the distance $\left\|u^{N}(T, \cdot)-u_{p}(T, \cdot)\right\|_{2}$. If the conjecture is correct, we expect the minimum to be attained for a function $p$ which is close to the predicted correction term $h^{\prime \prime}$. The result of a simulation is shown in figure 4. The figure shows that there is indeed a good fit between the conjectured and numerically determined correction terms. 

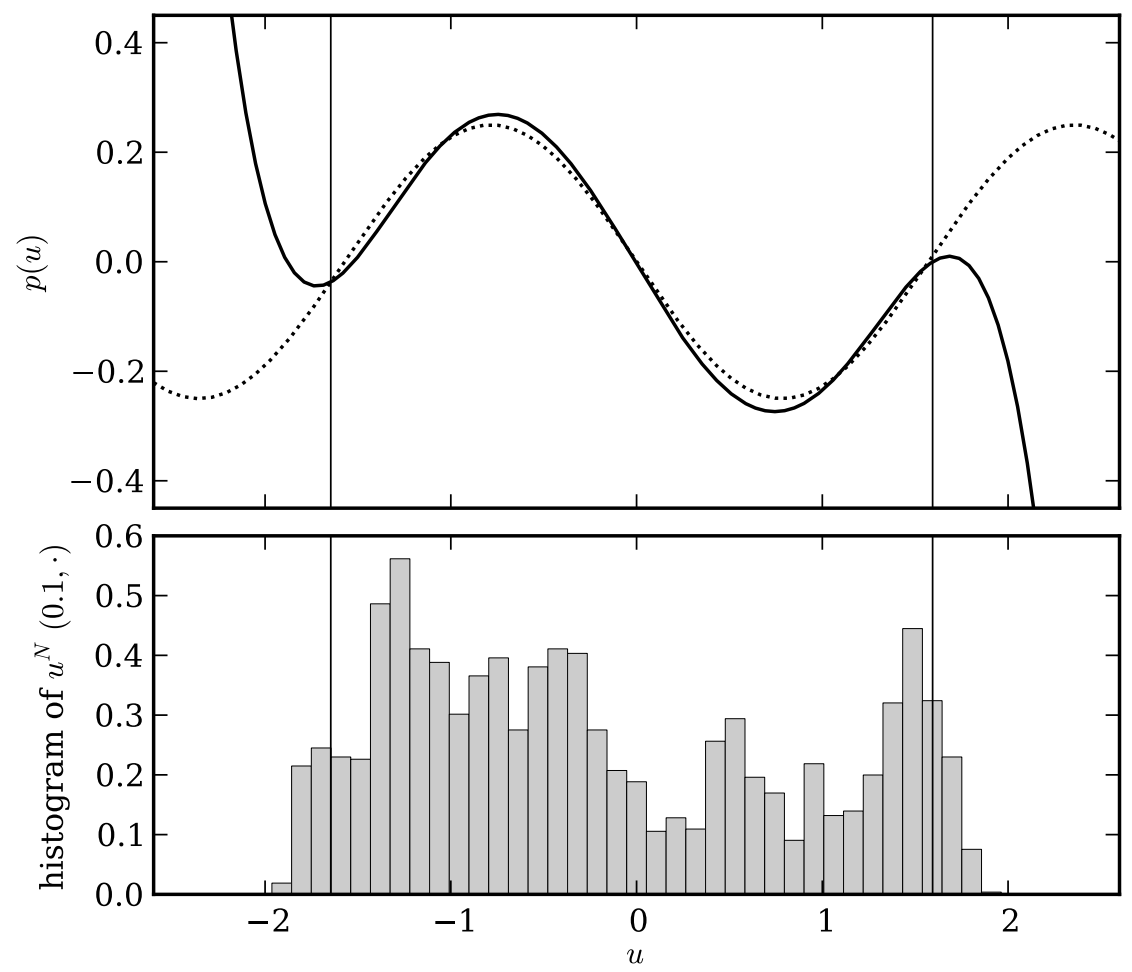

Figure 4: Illustration of the convergence of (22) to (23) for the one-dimensional example $h^{\prime}(x)=$ $\sin (x)^{2}$. For the figure we numerically compute the finite difference solution $u^{N}$ to (22). We then compute solutions $u_{p}$ to (24), using a fifth-order polynomial for the correction term $p$. This polynomial is then numerically fitted to minimise $\left\|u^{N}(1, \cdot)-u_{p}(1, \cdot)\right\|_{2}$. The top panel shows the resulting fitted correction term $-\sigma^{2} p / 4 \nu$ (full line) together with the correction term $-\sigma^{2} h^{\prime \prime}(u) / 4 \nu$ predicted in conjecture 3 (dotted line). To give an idea which range of the correction term is actually used in the computation, the lower panel shows the histogram of the values of $u^{N}$ (the vertical bars indicate the $5 \%$ and $95 \%$ quantiles). Between these quantiles, the graph shows a good fit between the numerically determined and conjectured correction terms. 


\subsection{Classically Ill-Posed Equations}

Pushing further the class of equations considered in the previous subsection, one may want to consider equations of the form

$$
d u_{i}=\nu \partial_{x}^{2} u_{i} d t+\sum_{j=1}^{d} G_{i j}(u) \partial_{x} u_{j} d t+f(u) d t+\sigma d w_{i}
$$

for some functions $G: \mathbb{R}^{d} \rightarrow \mathbb{R}^{d \times d}$ and $f: \mathbb{R}^{d} \rightarrow \mathbb{R}^{d}$. If we do not assume that $G$ has an antiderivative and since solutions are only expected to be $\alpha$-Hölder continuous in space for $\alpha<\frac{1}{2}$, it is no longer even clear what it means to be a solution to this equation. So, at least classically, (25) is ill-posed and the mere concept of solutions to such an evolution equation is difficult to establish.

However, the discretised equation does of course still make sense for any fixed value of $\varepsilon$. Furthermore, we observed numerically that there seems to be no instability as $\varepsilon \rightarrow 0$; indeed one observes pathwise convergence to a limiting process. By analogy with the behaviour observed for the situations where (25) is classically well-posed (i.e. when $G$ has an antiderivative), one would then be tempted to define solutions to (25) to be those processes that can be obtained as limits as $\varepsilon \rightarrow 0$ of the solutions to the equation where $\partial_{x} u_{j}$ is replaced by its symmetric discretisation. In analogy with conjecture 3 , we would then expect solutions of the right-sided discretisation to converge, as $\varepsilon \rightarrow 0$, to solutions of the corrected equation

$$
d u_{i}=\nu \partial_{x}^{2} u_{i} d t+\sum_{j}\left(G_{i j}(u) \partial_{x} u_{j}-\frac{\sigma^{2}}{4 \nu} \partial_{j} G_{i j}(u)\right) d t+f(u) d t+\sigma d w_{i}
$$

This reasoning leads to:

Conjecture 4 As $\varepsilon \rightarrow 0$, the equations

$$
d u_{i}^{\varepsilon}=\nu \partial_{x}^{2} u_{i}^{\varepsilon} d t+\sum_{j=1}^{d} G_{i j}\left(u^{\varepsilon}\right) D_{\varepsilon}^{1,0} u_{j}^{\varepsilon} d t+f\left(u^{\varepsilon}\right) d t+\sigma d w_{i}
$$

where $D_{\varepsilon}^{1,0}$ denotes the right-sided discretisation, and

$$
d \tilde{u}_{i}^{\varepsilon}=\nu \partial_{x}^{2} \tilde{u}_{i}^{\varepsilon} d t+\sum_{j=1}^{d}\left(G_{i j}\left(\tilde{u}^{\varepsilon}\right) D_{\varepsilon}^{1,1} \tilde{u}_{j}^{\varepsilon}-\frac{\sigma^{2}}{4 \nu} \partial_{j} G_{i j}\left(\tilde{u}^{\varepsilon}\right)\right) d t+f\left(\tilde{u}^{\varepsilon}\right) d t+\sigma d w_{i}
$$

where $D_{\varepsilon}^{1,1}$ denotes centred discretisation, converge to the same limit.

To test conjecture 4, we use the following numerical experiment: as an example we consider the SPDE

$$
\begin{aligned}
\partial_{t} u=\frac{1}{\sigma^{2}} \partial_{x}^{2} u+\frac{2}{\sigma^{2}}\left(\begin{array}{cc}
0 & \cos \left(u_{2}\right)-\sin \left(u_{1}\right) \\
\sin \left(u_{1}\right)-\cos \left(u_{2}\right) & 0
\end{array}\right) \partial_{x} u \\
+\frac{4}{\sigma^{2}}\left(\begin{array}{r}
\sin \left(u_{1}\right) \cos \left(u_{1}\right) \\
-\cos \left(u_{2}\right) \sin \left(u_{2}\right)
\end{array}\right)+\sqrt{2} \partial_{t} w .
\end{aligned}
$$

This SPDE is of the form (25) where $G$ has no antiderivative. SPDEs like (28) occur in the problem described in [HSV07, section 9] where we argue that the stationary distribution of this SPDE on $L^{2}\left([0,2 \pi], \mathbb{R}^{2}\right)$ (when equipped with appropriate boundary conditions) coincides with the distribution of the stochastic differential equation

$$
d U(t)=2\left(\begin{array}{r}
-\sin \left(U_{2}(t)\right) \\
\cos \left(U_{1}(t)\right)
\end{array}\right) d t+\sigma d B(t)
$$

For our experiment we numerically solve the SPDEs (26) and (27) and compare the solutions. The result is displayed in figure 5. As can be seen from the figure, the simulation results are in good agreement with conjecture 4. 

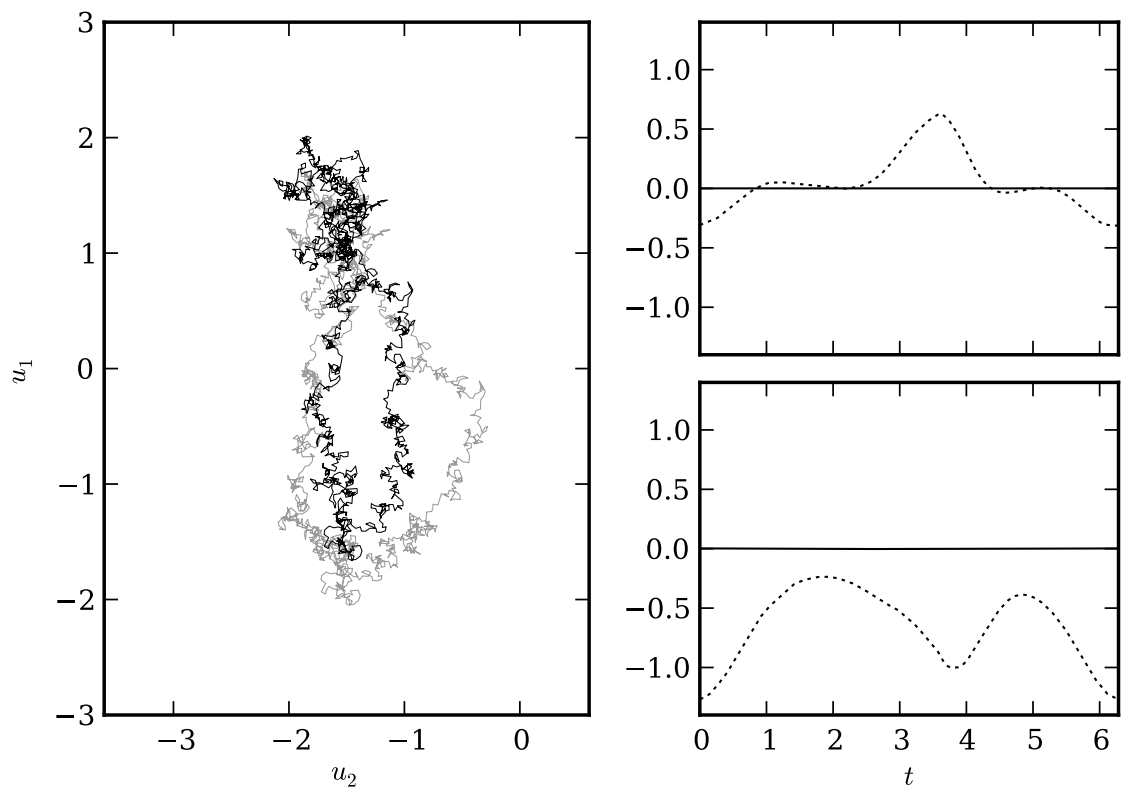

Figure 5: Illustration of the convergence in conjecture 4. The left-hand panel shows a numerical solution of the $\mathbb{R}^{2}$-valued SPDE (28) at a fixed timet. Since we use periodic boundary conditions, the plotted graph of $u(t, \cdot)$ forms a loop in $\mathbb{R}^{2}$. The black line in this plot was obtained using a centred discretisation, whereas the grey line in the background was obtained using a right-sided discretisation. As in figure 1, there is an $O(1)$ difference between the two discretisation schemes. The two plots on the right show the differences $u_{1}^{\varepsilon}-\tilde{u}_{1}^{\varepsilon}$ (upper panel) and $u_{2}^{\varepsilon}-\tilde{u}_{2}^{\varepsilon}$ (lower panel) between the discretisation schemes (26) and (27) from conjecture 4. (full lines). For comparison, the plots also show the differences $u_{1}^{\varepsilon}-\bar{u}_{1}^{\varepsilon}$ and $u_{2}^{\varepsilon}-\bar{u}_{2}^{\varepsilon}$ where $\bar{u}^{\varepsilon}$ is the solution of the uncorrected SPDE (28) using a centred discretisation (dotted lines). The graphs support conjecture 4 by showing good agreement between the solutions of (26) and (27). 


\subsection{Multiplicative Noise}

We conclude this section by considering the equation

$$
d u=\nu \partial_{x}^{2} u d t+g(u) \partial_{x} u d t+f(u) d w,
$$

where $g$ is as before and $f$ is a smooth bounded function with bounded derivatives of all orders. Such an equation is well-posed if the stochastic integral is interpreted in the Itô sense GN99. (Note that it is not well-posed if the stochastic integral is interpreted in the Stratonovich sense. This follows from the fact that, at least formally, the Itô correction term is infinite when $f$ is not constant.)

In such a case, the local quadratic variation of the solution is expected to be proportional to $f^{2}(u)$, so that one expects the right-sided discretisation to exhibit a correction term proportional to $g^{\prime}(u) f^{2}(u)$. More precisely, in analogy to conjecture 3, one would expect the following statement to hold.

Conjecture 5 The solution of

$$
d u=\nu \partial_{x}^{2} u d t+g(u) D_{\varepsilon} u d t+f(u) d w
$$

converges, as $\varepsilon \rightarrow 0$, to the solution of

$$
d u=\nu \partial_{x}^{2} u d t+g(u) \partial_{x} u d t-\frac{1}{4 \nu} g^{\prime}(u) f^{2}(u) d t+f(u) d w .
$$

To test this conjecture we perform a numerical experiment, similar to the one for conjecture 3 the result is shown in figure 6. The fit between predicted and numerically determined correction term in figure 6 is worse than in figure 4 and thus the numerical test is not entirely conclusive.

One possible reason is that the spatial resolution of our numerical simulations may not be sufficient. Indeed, the argument of the previous sections is based on a spatial averaging of the small-scale fluctuations of the process. In the case of multiplicative noise, these smallscale fluctuations are themselves multiplied by a the process $f(u)$, which is spatially quite rough. Therefore, this spatial averaging will hold only on extremely small scales, where $f(u)$ is essentially constant. In order to be seen by the numerical simulation, these scales still need to be resolved at sufficient precision to have some version of the law of large numbers.

\section{Small Noise/Viscosity Limit}

One regime that is of particular interest is the small noise/small viscosity limit. If one takes $\nu \propto \sigma^{2}$ in conjectures [1 and 2, one obtains a non-vanishing correction term even for arbitrarily small $\nu$ and $\sigma$ ! It is therefore of interest to study approximations to

$$
d u=\varepsilon \partial_{x}^{2} u d t-u \partial_{x} u d t+\sqrt{\varepsilon} d w
$$

for $\varepsilon \ll 1$.

It is well-known that, in the limiting case $\varepsilon=0$, finite difference schemes for the Burgers equations can only be used with extreme caution due to the presence of shocks in the solution. These shocks are jump discontinuities of the solution. The values $u^{-}, u^{+}$to the left/right of the jumps satisfy $u^{-}>u^{+}$; in other words, the jumps are always downwards jumps. For viscosity solution: 2 to the inviscid Burgers equation, shocks move through the system at velocity $\frac{1}{2}\left(u^{+}+u^{-}\right)$.

What happens at the formation of a shock? If the limiting non-viscous Burgers equation is discretised as

$$
\partial_{t} u_{n}=-u_{n} \frac{u_{n+1}-u_{n}}{\delta}
$$

we expect the discretisation scheme to be stable only when the discretisation is upwind in the sense that the direction of the discretisation coincides with the direction of propagation of the

\footnotetext{
${ }^{2}$ Also called entropy solutions, these are the solutions that are obtained as limits of (32) as $\varepsilon \rightarrow 0$
} 


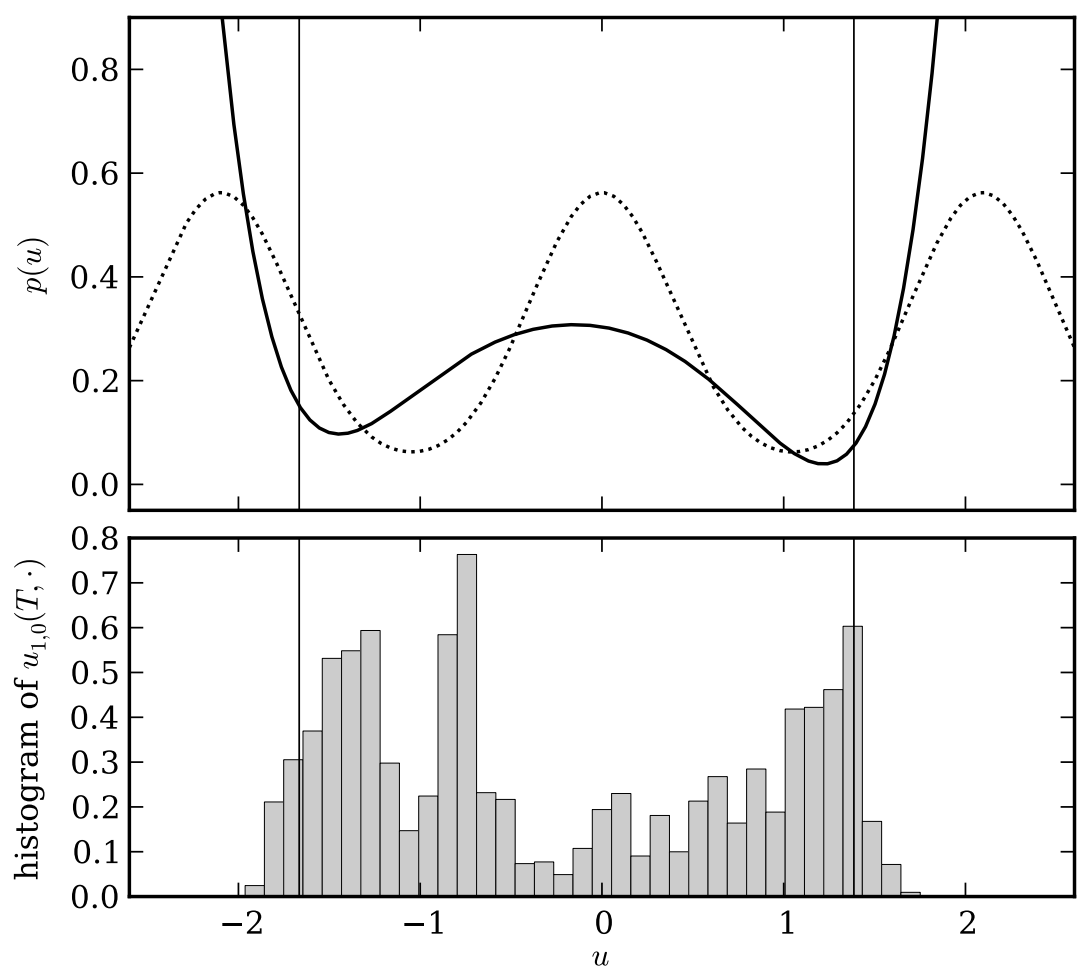

Figure 6: Illustration of the convergence of (30) to (31) in conjecture [5, for the case $g(u)=-u$ and $f(u)=1+\frac{1}{2} \cos (3 u)$. See figure 4 for an explanation of the graphs. Here we use a sixth-order polynomial $p$ to fit the correction term. The figure shows that the fit is significantly worse than in figure 因. See the text for a discussion of possible reasons for this effect. 
shock (see e.g. [CIR52, MRTB05]), but even in this case we expect the shock to propagate at the wrong speed.

Another problem is the following one: In the case of a non-conservative discretisation of the type (33), a simple linear stability analysis reveals that the discretised solutions develop an ultraviolet instability (i.e. the mode $v_{n}=(-1)^{n}$ becomes unstable) in the regions where $u>0$. Similarly, the corresponding left-sided discretisation shows an instability in the regions with $u<0$. In contrast, for the case of a centred discretisation, the highly oscillatory modes are stable independently of the sign of $u$.

Remark 4.1 Conservative discretisations, i.e.

$$
\partial_{t} u_{n}=-\frac{1}{2 \delta}\left(u_{n+1}^{2}-u_{n}^{2}\right),
$$

or variants thereof, still require the scheme to be upwind for stability, but in this case the shocks of the discretised system propagate at the correct speed. Also, these schemes do not suffer from the ultraviolet instability.

How is this picture modified for non-zero values of $\varepsilon$ ? The instabilities discussed above grow at a speed $\mathcal{O}\left(\delta^{-1}\right)$ while the stabilising effect of the viscosity is of the order $\varepsilon \delta^{-2}$. Thus, one expects the viscosity to dominate only if $\varepsilon \gg \delta$. Regarding the behaviour after the formation of a shock, a simple boundary layer analysis shows that a typical shock for (32) has width $\mathcal{O}(\varepsilon)$, so that the caveats pointed out above are expected to become relevant as soon as $\varepsilon \lesssim \delta$ (see for example EVE00a for a more sophisticated boundary layer analysis that even goes to the next order in $\varepsilon$ ).

On the other hand, at least away from shocks, the analysis performed in section 2 holds as soon as $u$ can be approximated by the solution to the linearised equation at sufficiently small (but still much larger than $\delta$ ) spatial scales. The $k$ th mode presents spatial features on a lenghtscale of order $k^{-1}$ and the nonlinearity essentially propagates the solution through space at speeds of order 1. Thus, the timescale on which the $k$ th mode varies due to the nonlinear effects is expected to be of order $k^{-1}$. On the other hand, the relevant timescale of the linear part for this mode is $\left(\varepsilon k^{2}\right)^{-1}$ and we can conclude from this heuristic consideration that the linearised equation is a good approximation to the full solution for modes with $k \gg \varepsilon^{-1}$, i.e. on space scales much smaller than $\varepsilon$. Consequently, one again expects the results from section 2 to be relevant as long as $\varepsilon \gg \delta$. This leads to the following statements.

Conjecture 6 For $\varepsilon \ll 1$, we expect the solution to the finite difference approximation of (32) to show the following behaviour.

1. For $\delta \ll \varepsilon$, the discretised solution converges to the viscosity solution of

$$
\partial_{t} u=-\frac{1}{2} \partial_{x}\left(u^{2}\right)+\frac{c}{4},
$$

where $c \in\{1,0,-1\}$ depending on whether the discretisation is right-sided, centred, or left-sided. Neither of the instabilities discussed above occur.

2. For $\varepsilon \ll \delta$, both viscosity and the noise term become irrelevant; the solution behaves like the corresponding approximation to the inviscid Burgers equation. In particular, as long as there are no shocks and while solutions have the correct sign to prevent ultraviolet blow-up, we expect to converge to (34) with $c=0$. After the occurrence of a shock, one expects stability only if the scheme is upwind and, for discretisations of type (33), shocks will have the wrong propagation speed.

To test this conjecture we again perform a numerical experiment. Keeping in line with the topic of this article, we focus on studying how the presence of the extra $c / 4$ term in (34) is affected by the values $\varepsilon, \delta>0$. For our experiment, we first solve the limiting equation (34) numerically up to a time $t>0$, once with $c=0$ and once with $c=1$, to get states $u_{0}(t), u_{1}(t) \in L^{2}([0,2 \pi], \mathbb{R})$. Now, for given $\varepsilon, \delta>0$, we solve the right-sided finite difference discretisation (13) for (32) to get a solution $u_{\varepsilon, \delta}(t)$. According to conjecture 6 we expect $u_{\varepsilon, \delta}(t) \approx u_{1}$ for $\delta \ll \varepsilon \ll 1$ and $u_{\varepsilon, \delta}(t) \approx u_{0}$ for $\varepsilon \ll \delta$. 


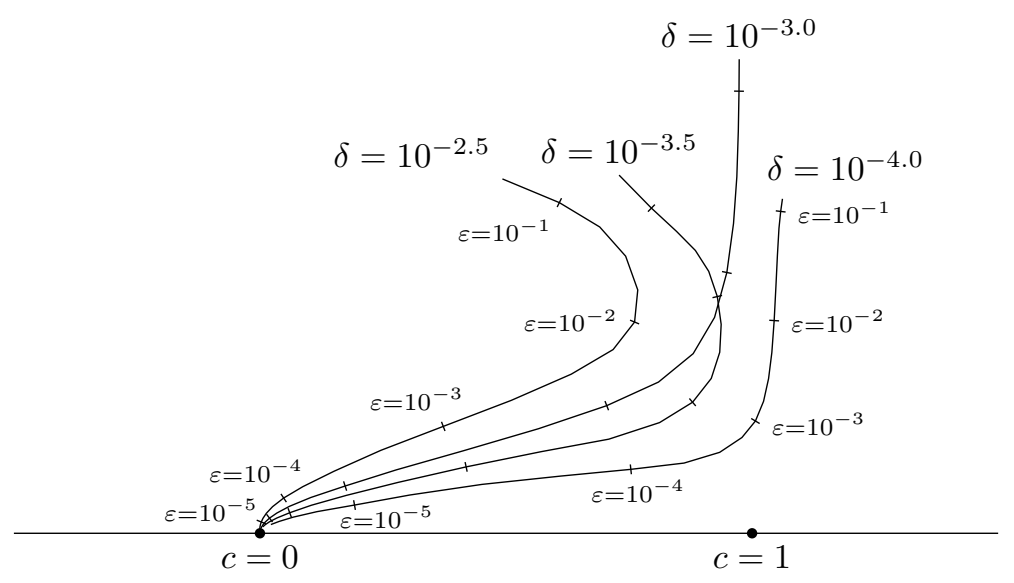

Figure 7: Illustration of the limiting behaviour of a right-sided finite difference discretisation for (32) as $\varepsilon \downarrow 0$ (for fixed $\delta$ ). Distances are shown in "two-centre bipolar coordinates" as described in the text. One can see that, as $\varepsilon$ gets small, the finite difference discretisation first gets close to the solution of (34) for $c=1$ but then finally converges to the solution with $c=0$ as $\varepsilon$ gets smaller than $\delta$. This behaviour reflects the dichotomy between the two cases of conjecture 6 .

To verify this conjecture, in figure 7 we consider, for fixed $\delta$, the solution $u_{\varepsilon, \delta}(t)$ as a function of $\varepsilon$. The coordinate system is chosen such that the (two dimensional) distance of $u_{\varepsilon, \delta}(t)$ to the point $c=0$ in the graph equals $\left\|u_{\varepsilon, \delta}(t)-u_{0}\right\|_{L^{2}}$; similarly the distance of $u_{\varepsilon, \delta}(t)$ to the point $c=1$ in the graph equals $\left\|u_{\varepsilon, \delta}(t)-u_{1}\right\|_{L^{2}}$ (this is sometimes called "two-centre bipolar coordinates"). The simulation indicates that the transition from $c=1$ to $c=0$ takes place at around $\varepsilon \approx \delta$ as expected.

\section{A Simulations}

To verify the heuristic arguments presented above, the text utilises a series of numerical results. This appendix summarises some of the technical aspects of these simulations 3

We first describe how to implement the finite difference schemes used to discretise SPDEs like (4), (21) and (29): For the space discretisation of these equations we approximate states $u \in L^{2}([0,2 \pi], \mathbb{R})$ by vectors $u^{N} \in \mathbb{R}^{N}$. The space discretisation of the differential operators given by formula (12), above. The finite difference discretisation of the white noise process $w$ is $W^{N} / \sqrt{\Delta x}$, where $W^{N}$ is a standard Brownian motion on $\mathbb{R}^{N}$ and $\Delta x=\delta=2 \pi / N$ is the space grid size. This leads to $\mathbb{R}^{N}$-valued stochastic differential equations of the form

$$
d u^{N}=\nu L_{N} u^{N} d t+F_{N}\left(u^{N}\right) d t+\sigma\left(u^{N}\right) \sqrt{\frac{1}{\Delta x}} d W^{N}(t)
$$

where $L_{N} \in \mathbb{R}^{N \times N}$ is the discretisation of the linear part and $F_{N}: \mathbb{R}^{N} \rightarrow \mathbb{R}^{N}$ is the discretisation of the nonlinearity.

For discretising time we use the $\theta$-method

$$
\begin{aligned}
u^{(n+1)}=u^{(n)}+ & \nu L_{N}\left(\theta u^{(n+1)}+(1-\theta) u^{(n)}\right) \Delta t \\
& +F_{N}\left(u^{(n)}\right) \Delta t+\sigma\left(u^{(n)}\right) \sqrt{\frac{\Delta t}{\Delta x}} \xi^{(n)}
\end{aligned}
$$

where $\Delta t>0$ is the time step size, $u^{(n)}$ is the discretised solution at time $n \Delta t$, the $\xi^{(n)}$ are i.i.d., $N$-dimensional standard normally distributed random variables, and $\theta=1 / 2$. Rearranging this

\footnotetext{
${ }^{3}$ For full details we refer to the source code of the programs used in these simulations, which is available for download at http://seehuhn.de/programs/HairerVoss10.
} 
equation gives

$$
\begin{aligned}
\left(I-\nu \theta \Delta t L_{N}\right) u^{(n+1)}=(I+\nu & \left.(1-\theta) \Delta t L_{N}\right) u^{(n)} \\
& +F_{N}\left(u^{(n)}\right) \Delta t+\sigma\left(u^{(n)}\right) \sqrt{\frac{\Delta t}{\Delta x}} \xi^{(n)} .
\end{aligned}
$$

Relation (35) allows to compute $u^{(n+1)}$ from $u^{(n)}$; since $I-\nu \theta \Delta t L_{N}$ is cyclic tridiagonal, this system can be solved efficiently.

Since we are using the partially implicit $\theta$-method for the linear part of the SDE, there are no constraints on the time step size $\Delta t$ arising from this term; on the other hand, since the non-linear transport term is treated explicitly, one has to make sure that $v \Delta t / \Delta x<C$, where $v$ is the largest speed of propagation appearing in the solution and $C$ is the Courant number, thus ensuring that the CFL condition is satisfied. The resulting method can be used to perform the simulations required to generate figures 1 5 and 7, as well as the "finite difference" curve in figure 2

As described in section 2 we can compute a spectral Galerkin approximation to the second order differential operator using discrete Fourier transform. This corresponds to replacing $L_{N}$ in (35) with

$$
\tilde{L}_{N}=\mathcal{F}^{-1} D \mathcal{F}
$$

where $D=\operatorname{diag}\left(-0^{2},-1^{2}, \ldots,-\lfloor N / 2\rfloor^{2}\right)$ and $\mathcal{F}: \mathbb{R}^{N} \rightarrow \mathbb{C}^{\lfloor N / 2\rfloor+1}$ represents the discrete Fourier transform (since the data is real, only $\lfloor N / 2\rfloor+1$ of the Fourier coefficients need to be considered). Because the matrix $\tilde{L}_{N}$ is no longer tridiagonal, one should not try to explicitly construct this matrix. Instead one can use the fact that $\mathcal{F}$ and $\mathcal{F}^{-1}$ can be computed efficiently: we can compute the right-hand side of (35) using

$$
\left(I+\nu(1-\theta) \Delta t L_{N}\right) u^{(n)}=\mathcal{F}^{-1} \operatorname{diag}\left(1-\nu(1-\theta) \Delta t k^{2}, k=0, \ldots,\lfloor N / 2\rfloor^{2}\right) \mathcal{F} u^{(n)}
$$

and to solve (35) for $u^{(n+1)}$ we can use the relation

$$
\left(I-\nu \theta \Delta t L_{N}\right)^{-1} b=\mathcal{F}^{-1} \operatorname{diag}\left(\frac{1}{1+\nu \theta \Delta t k^{2}}, k=0, \ldots,\lfloor N / 2\rfloor^{2}\right) \mathcal{F} b .
$$

This technique allows to obtain the "Galerkin" curve in figure 2 Similarly, the rougher-thanwhite noise for figure 3 was obtained by replacing the noise term $\xi^{(n)}$ with

$$
\tilde{\xi}^{(n)}=\mathcal{F}^{-1} \operatorname{diag}\left(\left(1+k^{2}\right)^{-\delta}, k=0, \ldots,\lfloor N / 2\rfloor\right) \mathcal{F} \xi^{(n)} .
$$

Finally, for the minimisation procedure performed to generate figures 4 and 6 we employ the simplex algorithm by Nelder and Mead [NM65].

\section{References}

[AG06] A. Alabert and I. Gyöngy. On numerical approximation of stochastic Burgers' equation. In From stochastic calculus to mathematical finance, pp. 1-15. Springer, Berlin, 2006 .

[Bar96] A.-L. Barabási. Roughening of growing surfaces: Kinetic models and continuum theories. Computational Materials Science, vol. 6, no. 2, pp. 127-134, 1996. doi: 10.1016/0927-0256(96)00026-2. Proceedings of the Workshop on Virtual Molecular Beam Epitaxy.

[BCF91] Z. Brzeźniak, M. Capiński and F. Flandoli. Stochastic partial differential equations and turbulence. Math. Models Methods Appl. Sci., vol. 1, no. 1, pp. 41-59, 1991. doi:10.1142/S0218202591000046.

[BCJL94] L. Bertini, N. Cancrini and G. Jona-Lasinio. The stochastic Burgers equation. Comm. Math. Phys., vol. 165, no. 2, pp. 211-232, 1994. 
$\left[\mathrm{BFG}^{+} 94\right]$ G. Blatter, M. V. Feigel'man, V. B. Geshkenbein, A. I. Larkin and V. M. Vinokur. Vortices in high-temperature superconductors. Rev. Mod. Phys., vol. 66, no. 4, pp. 1125-1388, 1994. doi:10.1103/RevModPhys.66.1125.

[BG97] L. Bertini and G. Giacomin. Stochastic Burgers and KPZ equations from particle systems. Comm. Math. Phys., vol. 183, no. 3, pp. 571-607, 1997. doi:10.1007/ s002200050044.

[BM96] J.-P. Bouchaud and M. Mézard. Velocity fluctuations in forced Burgers turbulence. Phys. Rev. E, vol. 54, no. 5, pp. 5116-5121, 1996. doi:10.1103/PhysRevE.54.5116.

[BQS09] M. Balazs, J. Quastel and T. Seppalainen. Scaling exponent for the Hopf-Cole solution of KPZ/Stochastic Burgers, 2009. Preprint. http://arxiv.org/abs/0909.4816

[CIR52] R. Courant, E. Isaacson and M. Rees. On the solution of nonlinear hyperbolic differential equations by finite differences. Comm. Pure. Appl. Math., vol. 5, pp. 243-255, 1952.

[DPDT94] G. Da Prato, A. Debussche and R. Temam. Stochastic Burgers' equation. NoDEA Nonlinear Differential Equations Appl., vol. 1, no. 4, pp. 389-402, 1994. doi:10.1007/ BF01194987.

[DPZ92] G. Da Prato and J. Zabczyk. Stochastic Equations in Infinite Dimensions, vol. 44 of Encyclopedia of Mathematics and its Applications. Cambridge University Press, 1992. ISBN 0-521-38529-6.

[EKMS00] W. E, K. Khanin, A. Mazel and Y. Sinai. Invariant measures for Burgers equation with stochastic forcing. Ann. of Math. (2), vol. 151, no. 3, pp. 877-960, 2000.

[EVE00a] W. E and E. Vanden Eijnden. Another note on forced Burgers turbulence. Phys. Fluids, vol. 12, no. 1, pp. 149-154, 2000.

[EVE00b] W. E and E. Vanden Eijnden. Statistical theory for the stochastic Burgers equation in the inviscid limit. Comm. Pure Appl. Math., vol. 53, no. 7, pp. 852-901, 2000.

[GD04] C. Gugg and J. Duan. A Markov jump process approximation of the stochastic Burgers equation. Stoch. Dyn., vol. 4, no. 2, pp. 245-264, 2004.

[GKN02] C. Gugg, H. Kielhöfer and M. Niggemann. On the Approximation of the Stochastic Burgers Equation. Communications in Mathematical Physics, vol. 230, no. 1, pp. 181-199, 2002.

[GM05] B. Goldys and B. Maslowski. Exponential ergodicity for stochastic Burgers and 2D Navier-Stokes equations. J. Funct. Anal., vol. 226, no. 1, pp. 230-255, 2005.

[GN99] I. Gyöngy and D. Nualart. On the stochastic Burgers' equation in the real line. Ann. Probab., vol. 27, no. 2, pp. 782-802, 1999.

[GSS85] S. N. Gurbatov, A. I. Saichev and S. F. Shandarin. A model description of the development of the large-scale structure of the Universe. Dokl. Akad. Nauk SSSR, vol. 285 , no. 2, pp. 323-326, 1985 .

[Gyö98] I. Gyöngy. Existence and uniqueness results for semilinear stochastic partial differential equations. Stochastic Process. Appl., vol. 73, no. 2, pp. 271-299, 1998.

[Hai09] M. Hairer. An Introduction to Stochastic PDEs. Lecture notes, 2009. http://arxiv.org/abs/0907.4178

[HSV07] M. Hairer, A. M. Stuart and J. Voss. Analysis of SPDEs Arising in Path Sampling, Part II: The Nonlinear Case. Annals of Applied Probability, vol. 17, no. 5, pp. 1657-1706, 2007. doi:10.1214/07-AAP441. 
[Kim06] J. U. Kim. On the stochastic Burgers equation with a polynomial nonlinearity in the real line. Discrete Contin. Dyn. Syst. Ser. B, vol. 6, no. 4, pp. 835-866 (electronic), 2006.

[Kra99] R. H. Kraichnan. Note on forced Burgers turbulence. Phys. Fluids, vol. 11, no. 12, pp. 3738-3742, 1999.

[MRTB05] R. M. M. Mattheij, S. W. Rienstra and J. H. M. ten Thije Boonkkamp. Partial Differential Equations. SIAM Monographs on Mathematical Modeling and Computation. Society for Industrial and Applied Mathematics (SIAM), 2005. ISBN 0-89871-594-6.

[NM65] J. Nelder and R. Mead. A Simplex Method for Function Minimization. Computer Journal, vol. 7, pp. 308-313, 1965.

[SZ89] S. F. Shandarin and Y. B. Zel'dovich. The large-scale structure of the universe: turbulence, intermittency, structures in a self-gravitating medium. Rev. Modern Phys., vol. 61, no. 2, pp. 185-220, 1989.

[TZ06] K. Twardowska and J. Zabczyk. Qualitative properties of solutions to stochastic Burgers' system of equations. In Stochastic partial differential equations and applications - VII, vol. 245 of Lect. Notes Pure Appl. Math., pp. 311-322. Chapman \& Hall/CRC, Boca Raton, FL, 2006.

[YC96] V. Yakhot and A. Chekhlov. Algebraic Tails of Probability Density Functions in the Random-Force-Driven Burgers Turbulence. Phys. Rev. Lett., vol. 77, no. 15, pp. 3118-3121, 1996. doi:10.1103/PhysRevLett.77.3118. 